\title{
Precursor of Plasmacytoid Dendritic Cell
}

National Cancer Institute

\section{Source}

National Cancer Institute. Precursor of Plasmacytoid Dendritic Cell. NCI Thesaurus. Code C45236.

A cell found in peripheral blood, lymphoid tissue, and some inflamed tissue. It produces vast amounts of type I interferons especially in response to viruses, therefore playing an important role in antiviral immunity and triggers for adaptive immune responses. 